\title{
Previsão do Consumo de Energia Elétrica na Região Nordeste do Brasil
}

\author{
Electricity consumption forecasting in Brazilian northeastern region
}

Iván Patricio Moreno Marcos ${ }^{1}$ (i) orcid.org/0000-0002-0631-8488

\author{
Armando Pereira Pontes Júnior 1 (D) orcid.org/0000-0002-8212-4589 \\ ${ }^{1}$ Escola Politécnica de Pernambuco, Universidade de Pernambuco, Recife, Brasil. \\ E-mail do autor principal: Iván Patricio Moreno Marcos ipmm@ecomp.poli.br
}

\section{Resumo}

Sistemas elétricos de potência coletam grandes volumes de dados que podem fornecer informações valiosas sobre o consumo de energia. As empresas elétricas podem usar esses dados históricos de consumo para auxílio no processo de tomada de decisões no que diz respeito ao planejamento da produção de energia, principalmente pelo lado da demanda (consumo de energia esperado). Neste trabalho o problema de previsão de consumo de energia foi modelado como sendo univariado com um passo temporal à frente. Foram utilizados os algoritmos Naive (Persistente, Média e Mediana), SARIMA, MLP, CNN e LSTM; e foi desempenhada uma busca gulosa de seus hiperparâmetros com o objetivo de encontrar a melhor configuração associada a cada algoritmo. Além disso, para comparação e escolha do melhor algoritmo de previsão foi utilizado a métrica MAPE e o teste de hipótese de Deibold-Mariano modificado. Para a prova de conceito da proposta metodológica, foram usados dados de consumo de energia da região Nordeste do Brasil entre os anos de 2004 a 2019.

Palavras-Chave: MLP; CNN; LSTM; SARIMA; Previsão do Consumo de Energia; Aprendizagem de Máquina.

\begin{abstract}
Electric power systems collect large volumes of data that can provide valuable information on energy consumption. Electric utilities can use this historical consumption data to assist in the decision-making process with regard to energy production, through estimating the expected energy consumption. In this work, the energy consumption forecasting problem was modeled as being univariate with a temporal step forward. The algorithms: Naive (Persistent, Mean and Median), SARIMA, MLP, CNN and LSTM were used; and a greedy search of its hyperparameters was performed in order to find the best configuration associated with each algorithm. In addition, for comparison and choice of the best forecasting algorithm, the MAPE metric and the modified Deibold-Mariano hypothesis test were used. For the proof of concept of the methodological proposal, energy consumption data from the Northeast region of Brazil between the years 2004 to 2019 were used.
\end{abstract}

Key-words: MLP; CNN; LSTM; SARIMA; Energy Consumption Forecasting; Machine Learning. 


\section{Introdução}

A energia é um insumo basilar nas economias modernas. A história do desenvolvimento econômico e social de todos os países está intimamente relacionado ao desenvolvimento e a expansão da oferta de energia elétrica. De acordo com a Empresa de Pesquisa Energética - EPE, a matriz elétrica do Brasil é baseada principalmente em fontes renováveis, sendo a hidráulica a principal fonte de geração de energia elétrica $(65,2 \%)$, seguida por gás natural $(10,5 \%)$, Biomassa $(8,2 \%)$, energia Solar e Eólica $(6,9 \%)$, Carvão $(4,1 \%)$, energia Nuclear $(2,6 \%)$ e Petróleo e Derivados (2,5\%) [1].

A geração de energia elétrica ocorre em ambiente de livre concorrência com a venda da energia por meio de leilões ou de livre negociação. Geralmente os contratos de compra e venda de energia são de longo prazo, o que minimiza o risco inerente do setor.

O principal sistema de transmissão nacional é todo integrado (conhecido como SIN - Sistema Integrado Nacional) e visa ligar as diversas regiões do país através de subsistemas: Sul, SudesteCentro-Oeste, Nordeste e Norte.

Especificamente sobre Subsistema Nordeste, a energia elétrica que era produzida provinha basicamente da fonte hídrica - com grande importância das águas do Rio São Francisco. Em tempos mais recentes, este cenário vem se modificando com a abertura para outras fontes de geração de energia elétrica. Destaca-se o crescimento expressivo e do aumento da capacidade instalada da geração eólica, muito por reflexo de ser uma via alternativa as baixas precipitações pluviométricas que a região vivencia e pela quantidade de dias ensolarados que a região experimenta. Dados, na posição de fevereiro/2019, revelam que a fonte eólica já lidera a matriz de energia elétrica do Nordeste com participação de $35,46 \%$, seguida da fonte hidráulica, com $32,49 \%$ [2].

Apesar do crescimento expressivo da fonte eólica, - Subsistema Nordeste ainda se mostra deficitário, sendo importador líquido de energia elétrica. Em 2018 este déficit de geração de energia elétrica chegou a 1.642 MW médios, o que equivalia a 15,2\% da carga [2]. Assim, faz-se necessário investigar como essas mudanças afetam a demanda por energia elétrica na região Nordeste.
Essa pesquisa deriva do fato da energia elétrica não possuir uma viabilidade técnico-econômico de ser armazenada, sua produção e consumo devem ser cuidadosamente planejados de forma a evitar situações de insuficiência energética (falta de abastecimento), bem como de superprodução (desperdício). Desta forma, possuir uma ferramenta de previsão de demanda de energia é fundamental para auxiliar o processo de tomada de decisão e de investimentos por parte dos entes envolvidos.

\section{Materiais e métodos}

Nesta seção é apresentada os conjuntos de dados selecionados para o estudo, a metodologia para desempenhar a previsão de séries temporais usando modelos clássicos e de machine learning, e os detalhes do estudo experimental desempenhado.

\subsection{Operador Nacional do Sistema Elétrico (ONS)}

Após a definição do problema de negócio: "Previsão do consumo de energia elétrica na região Nordeste do Brasil" sendo modelado como um problema de previsão univariado um passo à frente deve-se identificar o Stakeholder que contém a serie a ser usada, neste caso o ONS.

O ONS contém diversas informações energia no território brasileiro. Para este trabalho foi usada a variável Carga de Energia (CE). Ela contém registros diários do consumo de energia da região Nordeste do Brasil no período compreendido entre 2004 a 2019, totalizando 5.844 observações.

\subsection{Análise descritiva dos dados do ONS}

A primeira análise que pode ser feita à variável CE é uma estatística. As 5.840 observações dão uma ideia de quantas observações podem ser usadas para treinamento e teste para um modelo de machine learning. A média de 8.559 aproximadamente seria linha base da série temporal. O desvio padrão dá uma ideia da distância dos valores em relação à média. Neste caso tem-se um alto desvio dos dados. Têm-se os percentis para elaborar o boxplot. Os valores máximo e mínimo que podem ser usados para normalizar a série temporal.

$\begin{array}{cc}\text { count } & 5840.000000 \\ \text { mean } & 8558.735315 \\ \text { std } & 1500.038727 \\ \text { min } & 5193.175417\end{array}$




$\begin{array}{lr}25 \% & 7279.704927 \\ 50 \% & 8507.408812 \\ 75 \% & 9858.647688 \\ \text { max } & 13536.630628\end{array}$

Pode-se elaborar um gráfico de linhas (Figura 1) para observar rapidamente algumas características como possíveis valores anómalos, componentes de tendência e sazonalidade. Assim, é possível observar claramente que existe uma tendência. Também é possível observar a presença de um provável outlier entre os anos 2014 - 2016. Também pode-se observar uma componente sazonal anual.

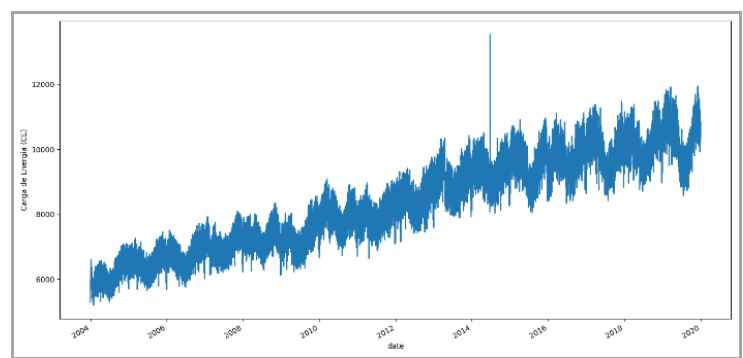

Figura 1. Gráfico de linhas para a variável CE.

Esta primeira análise é importante porque guiará a seleção do modelo linear (Ex. Modelo SARIMA). Também ditas componentes podem ser usados como séries de entrada para os modelos de machine learning (Ex. LSTM). E os possíveis outliers podem ser processados com alguma técnica de seleção de características (Ex. Random Forest). Esta fase de pré-processamento de dados também pode ser chamado de Engenharia de Características.

Uma outra visualização importante é a distribuição dos dados, o gráfico de densidade (Figura 2) é elaborado a partir do Histograma. Este é um gráfico sem ordem temporal. Trata-se de um gráfico importante já que alguns métodos de previsão de séries temporais lineares, como os modelos SARIMA, assumem uma distribuição de observações do tipo Normal ou Gaussiana. Na Figura 2 pode-se observar que certamente existem duas gaussianas superpostas ou uma Gaussiana Dupla.

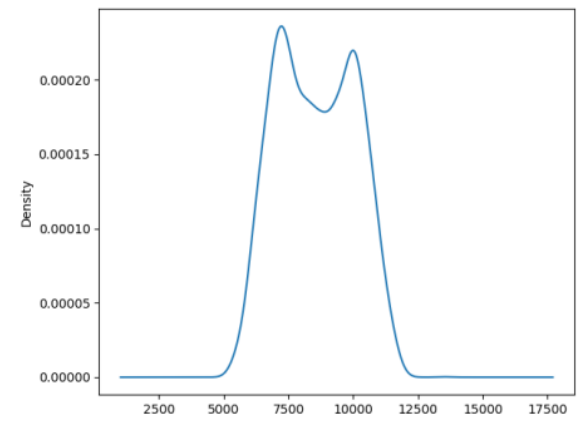

Figura 2. Gráfico de densidade para a variável CE.

Uma maneira de analisar a distribuição dos dados por intervalo é usar o gráfico de boxplot (Figura 3). Este tipo de gráfico utiliza os percentis calculados anteriormente para desenhar as caixas por ano. A linhas centrais das caixas representa a mediana desse conjunto anual de dados. É importante notar que os pontos fora das caixas são chamados de pontos exteriores (valores atípicos). Uma forma de processar esses dados é extrair os valores atípicos. Ou seja, entregar para o modelo sem esses valores atípicos e avaliar o desempenho do modelo.

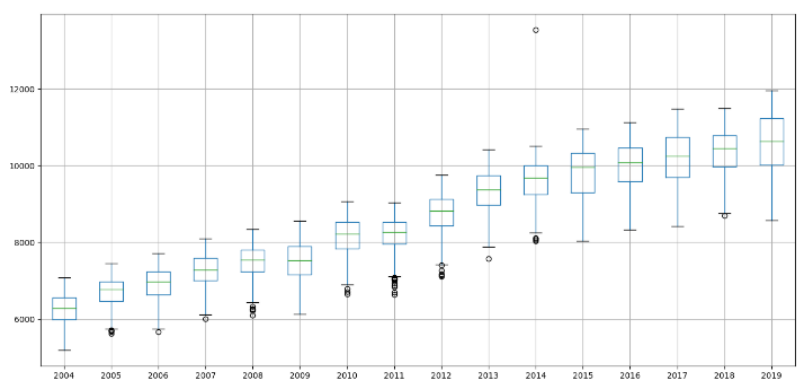

Figura 3. Gráfico Boxplot para a variável CE.

A modelagem de séries temporais assume uma relação entre uma observação e sua observação passada. Essas observações passadas são chamadas de lag. Podem-se criar gráficos de uma variável com seus lags (lag-1, lag- 2 , etc.) como mostrado na Figura 4. Analisando os gráficos para a variável CE e seus lags, pode-se inferir que existem uma correlação positiva. Em outras palavras, existe uma relação linear entre CE e seus valores passado até lag-7. Este tipo de gráfico poderia ser usado para determinar a ordem ' $\mathrm{p}$ ' de um modelo SARIMA, ou seja, $p=7$ (de tendência). 


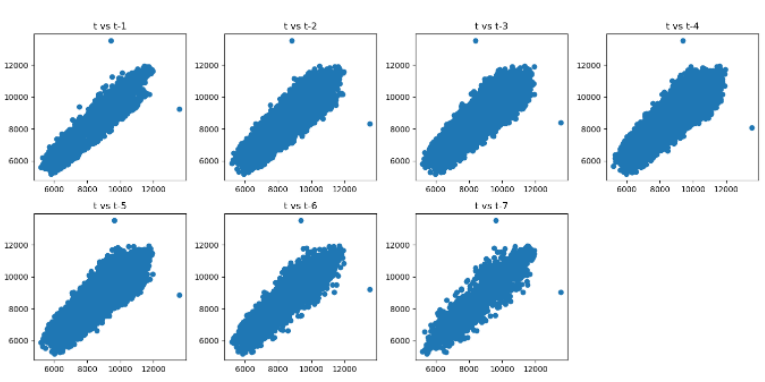

Figura 4. Gráfico de dispersão contendo lag-7 para a variável CE.

Existe outro tipo de gráfico chamado de autocorrelação que pode mensurar a relação linear de uma variável com seus lags. Este valor varia entre -1 e 1 , significando que o valor 1 representa uma forte correlação positiva e que o valor -1 uma forte correlação negativa. Já o valor 0 , representa a ausência de correlação. Na Figura 5, pode-se observar os graus de correlações lineares entre a variável CE e seus 6.000 primeiros lags, sugerindo que poderiam ser usados os primeiros 400 lags, aproximadamente. Este gráfico pode ser usado para selecionar quantos lags de cada variável podem ser selecionados tanto para modelos lineares como de machine learning.

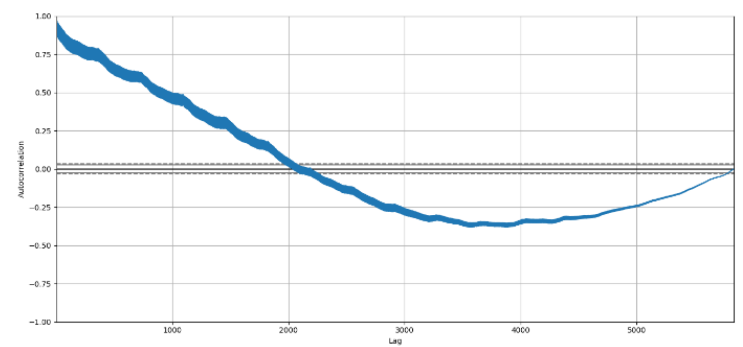

Figura 5. Gráfico de autocorrelação para a variável CE.

\subsection{Metodologia}

Nesta subseção são descritos os principais passos de pré-processamento e os conceitos fundamentais por trás dos modelos persistente, clássicos (ARIMA, SARIMA) e de redes neurais (MLP, CNN, LSTM) utilizadas.

\subsubsection{Pré-processamento dos dados}

Uma vez feito o download das tabelas temporais associadas ao problema de pesquisa, cada tabela contendo um conjunto de atributos, é necessário fazer o pré-processamento visando a adequação dos dados para serem as entradas dos modelos de previsão de séries temporais.

Lembrando que nesta fase se objetiva montar a tabela de treinamento e de teste para que o modelo clássico e de machine learning possam desempenhar a previsão da variável em estudo.

Cabe mencionar que todas as colunas que não foram usadas pelo modelo foram excluídas usando 0 Excel (Figura 6), resultando em tabelas basicamente com colunas temporal e de atributo (Figura 7). Por outro lado, o tratamento da coluna temporal foi formatado para o formato (YYYY-MM-DD), usando o Excel.

\begin{tabular}{|l|l|}
\hline \multicolumn{1}{|c}{ C } & \multicolumn{1}{c}{ D } \\
\hline Periodo Exibido CE Simp 4 & Subsistema \\
\hline J Dia & Nordeste \\
\hline J Dia & Nordeste \\
\hline J Dia & Nordeste \\
\hline J Dia & Nordeste \\
\hline J Dia & Nordeste \\
\hline J Dia & Nordeste \\
\hline J Dia & Nordeste \\
\hline J Dia & Nordeste \\
\hline J Dia & Nordeste \\
\hline J Dia & Nordeste \\
\hline J Dia & Nordeste \\
\hline J Dia & Nordeste \\
\hline Dia & Nordeste \\
\hline nin & ainrd rntr \\
\hline
\end{tabular}

Figura 6. Colunas com atributos contendo valores repetitivos associadas à tabela $\mathrm{CE}$.

\begin{tabular}{|c|c|c|}
\hline 4 & \multicolumn{1}{|c|}{ A } & \multicolumn{1}{c|}{ B } \\
\hline 1 & date & CE \\
\hline 2 & $2004-01-01$ & 5282,913333 \\
\hline 3 & $2004-01-02$ & 5975,419167 \\
\hline 4 & $2004-01-03$ & 5979,946667 \\
\hline 5 & $2004-01-04$ & 5664,687083 \\
\hline 6 & $2004-01-05$ & 6330,049167 \\
\hline 7 & $2004-01-06$ & 6352,937917 \\
\hline 8 & $2004-01-07$ & 6386,322917 \\
\hline 9 & $2004-01-08$ & 6603,959167 \\
\hline 10 & $2004-01-09$ & 6604,383750 \\
\hline
\end{tabular}

Figura 7. Formato final da tabela CE.

Um outro aspecto de transformação de dados diz respeito à normalização entre 0.10 e 0.90 . Neste quesito foi adotado a transformação linear seguindo a equação (1) e a Tabela 1 :

$$
y=\frac{(b-a)\left(x_{i}-x_{\min }\right)}{\left(x_{\max }-x_{\min }\right)}+a
$$

Onde $X_{\min }$ e $X_{\max }$ são os valores mínimo e máximo da série temporal associada à variável em questão.

Tabela 1. Valores adotados para normalização.

\begin{tabular}{l|l} 
b & 0,9
\end{tabular}




\section{a}

0,1

No caso da variável $\mathrm{CE}$, os seus valores antes e despois da normalização ficaram como mostrado na Figura 8.

\begin{tabular}{|c|c|c|c|}
\hline \multicolumn{2}{|c|}{ A } & \multicolumn{1}{c|}{ B } & C \\
\hline 1 & date & CE & CE_norm \\
\hline 2 & $2004-01-01$ & 5282,913333 & 0,108604389 \\
\hline 3 & $2004-01-02$ & 5975,419167 & 0,175004298 \\
\hline 4 & $2004-01-03$ & 5979,946667 & 0,175438411 \\
\hline 5 & $2004-01-04$ & 5664,687083 & 0,145210207 \\
\hline 6 & $2004-01-05$ & 6330,049167 & 0,209007477 \\
\hline 7 & $2004-01-06$ & 6352,937917 & 0,211202131 \\
\hline 8 & $2004-01-07$ & 6386,322917 & 0,214403203 \\
\hline 9 & $2004-01-08$ & 6603,959167 & 0,235270936 \\
\hline 10 & $2004-01-09$ & 6604,383750 & 0,235311647 \\
\hline 11 & $2004-01-10$ & 6214,840000 & 0,197960814 \\
\hline
\end{tabular}

Figura 8. Variável CE antes e despois da normalização.

\subsubsection{Modelos Naive}

Estas abordagens apenas proporcionam 0 baseline de desempenho (limiar inferior) associado ao problema. Desta forma, pode-se obter uma ideia de quão bom os demais modelos (mais sofisticados) se desempenham nesse problema [3]. Embora simples, eles podem ser ajustados a um determinado problema. Por exemplo, no modelo persistente definir a observação na qual vai persistir. Para a estratégia baseada em média ou mediada deve-se definir quantas observações passadas devem ser escolhidas. A sintonização destes hiperparâmetros podem prover um limite inferior mais robusto sobre o desempenho do modelo. Além disso, podem ajudar na escolha e configuração de métodos mais sofisticados.

\section{Estratégia Persistente}

Usa uma das observações passadas como valor de previsão. Pode ser ajustado para observações sazonais. Neste caso, a observação no mesmo tempo do ciclo (offset) anterior talvez seja usado. Pode-se generalizar para testar diferentes offsets. Não se faz tratamento nos dados.

\section{Estratégia baseada em Média ou Mediana}

Calcula a média ou mediana de um conjunto de observações prévias para logo ser usada como valor de previsão sem nenhum tratamento nos dados. 0 conjunto de observações pode ser fixado a um 25 número reduzidos das últimas observações passadas ou generalizado para testar cada possível ' $n$ ' conjuntos de observações passadas para calcular a média ou mediana.

\subsubsection{Modelos Autoregressivos ARIMA/SARIMA}

O modelo ARIMA (AutoRegressive Integrated Moving Average) é uma classe de método estatístico para analisar e fazer previsão de séries temporais. É uma generalização de um modelo mais simples chamado ARMA (AutoRegressive Moving Average), na qual é adicionada a noção de integração [3][4]. Os aspectos chaves do modelo podem ser descritos como segue [5]:

- AR: Autoregressão. Um modelo que usa a relação dependente entre uma observação e um número de observações defasadas;

- I: Integrado. O uso de diferenciação nas observações (subtraindo cada observação com sua observação prévia imediata) com o objetivo de tornar série temporal estacionária;

- MA: Médias Móveis. Um modelo que usa a dependência entre uma observação e um erro residual (calculado quando aplicado um modelo de médias móveis a observações defasadas).

Pode ser especificado como ARIMA ( $p, d, q)$, onde os parâmetros $\mathrm{p}$ (minúsculo, para a ordem autoregressiva tendencial), d (minúsculo, para a ordem de diferenciação tendencial) e q (minúsculo, para a ordem de médias móveis tendencial). Os parâmetros devem ser números inteiros e configuram o modelo. As componentes $A R$, I e MA, estão associadas aos parâmetros $p, d, q$ respectivamente. O modelo ARIMA não dá suporte a dados sazonais (séries temporais com período repetitivo). O modelo ARIMA espera dados não sazonais ou dados cuja componente sazonal foi removida usando diferenciação sazonal.

O processo ARIMA implica construir um modelo de regressão linear considerando uma ordem a cada parâmetro, e a séries temporal é tratada (por diferenciação) para garantir sua estacionaridade, o qual se não for feita, pode afetar o desempenho do modelo.

Por outro lado, um modelo SARIMA (Seasonal AutoRegressive Integrated Moving Average) é uma

DOI: $10 . x x x x / s 11468-014-9759-3$ 
extensão do modelo ARIMA que suporta dados sazonais. Ele adiciona mais 4 parâmetros, $P$ (maiúsculo, para a ordem autoregressiva sazonal), D (maiúsculo, para a ordem de diferenciação sazonal), Q (maiúsculo, para a ordem de médias móveis sazonal) e $m$ (número de passos para um único período sazonal).

Configurar tanto um modelo ARIMA quanto SARIMA implica atribuir valores aos parâmetros, tanto na ordem sazonal quanto na tendencial. Para notação do modelo SARIMA segue-se: $\operatorname{SARIMA}(p, d$, q) $(P, D, Q, t) m$. Os gráficos ACF (AutoCorrelation Function) e PACF (Partial AutoCorrelation Function) são usados para definir de forma aproximada os parâmetros do ARIMA. Na prática um esquema de grid search (teste de várias configurações de modelos SARIMA) é usado para estimar os parâmetros.

\subsubsection{Modelo MLP}

Trata-se de uma rede neural composta por camadas de neurônios que se ligam através de sinapses com pesos. O MLP (MultiLayer Perceptron) é formado por 3 tipos de camadas, uma de entrada, uma camada de saída e uma ou mais camadas ocultas (hidden layers), onde ocorrem os cálculos computacionais [6]. Visto que o MLP é uma rede plenamente conectada, os nós de cada camada se conectam imediatamente com os nós da camada posterior e estas conexões são ponderadas pelos pesos. O MLP é muito utilizado nos casos de problemas não lineares, onde um único hiperplano não consegue dar bons resultados. A lógica de treinamento de um MLP é por algoritmo de retropropagação de erro, que consiste em duas etapas: o processo direto e o processo reverso. Inicialmente não se possui informações sobre os pesos da rede, então, deve-se inicializar os pesos de forma aleatória com distribuição uniforme próximo de zero. Assim, no processo direto a entrada de valor é propagada pela rede e os pesos se mantém constantes. No processo reverso, um sinal de erro da saída é propagado no sentido contrário ao sinal de entrada e os pesos são corrigidos de acordo com algum regramento. Esse retroprocessamento é contínuo e mantém-se até que critérios de parada sejam atendidos.

\subsubsection{Modelo CNN}

Uma rede neural convolucional é uma arquitetura de rede neural de aprendizado profundo e que foi inspirada nos processos biológicos da visão. As conexões dos neurônios se baseiam na forma como o córtex visual dos animais funciona. Por isso que este tipo de rede é muito utilizado em problemas de reconhecimento de imagens. Uma das vantagens de se utilizar uma CNN (Convolutional Neural Network) é que o pré-processamento é menos custoso do que outros algoritmos de classificação.

Nas CNNs apenas subconjuntos de entradas são conectadas aos neurônios, o que é bem diferente das redes ditas plenamente conectadas, onde todos os neurônios estão conectados. Essa mudança de arquitetura faz com que se diminua o número de parâmetros a serem aprendidos e, consequentemente, há um ganho de tempo no processo de treinamento da rede [7].

\subsubsection{Modelo LSTM}

Rede neural desenhada para abordar problemas de sequência. Possui na sua arquitetura conexões com loops, adicionando assim retroalimentação e memória. Esta memória permite a rede aprender e generalizar através de sequências de entradas, ao invés de padrões individuais. Um tipo de rede neural recorrente é a rede LSTM (Long Short-Term Memory). A LSTM adquire potencial contrastado no estado da arte quando concebidas de forma empilhada. Existe uma limitação para o MLP para o caso de um problema de previsão de séries temporais univariado, que quando se define a janela de entradas para o MLP esta é fixa e deve ser escolhida com conhecimento baseado no contexto do problema. Em outras palavras, um tamanho de janela inadequado não capturaria padrões relevantes para fazer previsão univariado [8].

No que diz respeito ao seu funcionamento e arquitetura, um neurônio pode passar seu sinal para seus neurônios vizinhos além de passar o sinal para frente. A saída da rede pode ser conectada à entrada da rede recorrente. As conexões recorrentes adicionam um estado ou memória a rede, isto permite aprender uma maior extensão de abstrações a partir da sequência de entradas. A diferença das MLPs que possuem neurônios, as redes LSTMs possuem blocos de memória que são conectadas em camadas. Esses blocos contêm comportas que gerenciam o estado do bloco e sua saída. Uma unidade de memória opera sobre uma sequência de entrada e cada comporta dentro de cada unidade usa uma função de ativação sigmoide para controlar se eles são ativados ou não, fazendo uma mudança no 
seu estado e adicionando informação para fluir através da unidade condicional. O algoritmo de treinamento da RNN (Recurrent Neural Network) é chamado de Backpropagation Through Time (BPTT). Cada unidade de memória é como um mini estado de máquina onde as comportas da unidade possuem pesos que são aprendidos durante o treinamento. Toda LSTM possui os seguintes componentes nas suas unidades de memória:

- Forget gate: Decide que informação é descartada da unidade de memória;

- Input gate: Decide quais valores da entrada devem ser usados para atualizar o estado da memória;

- Output gate: Decide qual é a saída baseada na entrada e a memória da unidade.

No entanto, ainda existem os seguintes desafios:

- Como treinar uma RNN com backpropagation;

- Como lidar com os temas de VANISHING e EXPLODING durante treinamento.

\subsection{Estudo experimental}

Nesta subseção é apresentado o desenho do estudo experimental conduzido usando os dados associados à variável em estudo. Além disso, são descritas as configurações dos modelos para o processo de busca dos hiperparâmetros para cada arquitetura, a métrica de avaliação e o teste de hipótese.

O conjunto de dados do consumo de energia (Variável CE pertencente ao Stakeholder ONS) da região Nordeste para previsão de um dia a frente. Ao todo têm-se 5.844 observações, das quais, 5.479 observações serão usadas como treinamento e o restante, 365, como teste. Cabe dizer que para este cenário, serão apresentados os 3 melhores modelos com menor erro associado.

Para rodar os experimentos, usou-se um computador com processador Intel ${ }^{\circledR}$ Core $^{\mathrm{TM}}$ i7, CPU 2.2Ghz com 8.00GB RAM. Foi utilizada a linguagem Python 3.7 com o auxílio das seguintes bibliotecas: TensorFlow 2.0, Keras, SKlearn, StatsModels e SciPy.

\subsubsection{Modelos}

A seguir são expostos de maneira sucinta as principais configurações dos modelos apresentados no item 2.3.2 até o 2.3.6 para desempenhar a busca dos hiperparâmetros com o objetivo de testar vários modelos candidatos e escolher a configuração que atingir um menor MAPE (Mean Absolute Percentual Error).

\section{Modelo Simples}

Ao todo foram testadas 34.992 possíveis configurações baseadas em Persistente, Média e Mediana. Somado a isso, testou-se modelos simples usando componentes sazonais de 12 meses, já que, os dados usados de consumo apresentam um período sazonal de 12 meses ou 365 dias.

\section{Modelo SARIMA}

Para fins de simulação utilizou a seguinte configuração de SARIMA. A partir de um grid search foram testados um total de 2.592 possíveis configurações. As configurações dos parâmetros do modelo SARIMA foram: tendência $(p, d, q=[0,1$, 2]) e sazonal $(P, D, Q=[0,1,2])$, com período sazonal $\mathrm{m}=12 \mathrm{e}$ sem período sazonal $\mathrm{m}=0 \mathrm{e}$ quatro possíveis modelos de tendência [ ' $n$ ', ' $c$ ', ' $t$ ', 'ct'], significando: $n$ : sem tendência, c: constante, t: linear e ct: uma constante com tendência linear.

\section{Modelo MLP}

Para fins de simulação se fixou uma rede neural de uma camada oculta com função de ativação RELU, otimizador ADAM, função perda MSE (Mean Squared Error) e um neurônio na camada de saída já que, trata-se de um problema de previsão um passo à frente. Os hiperparâmetros que serão buscados no grid search são: número de entradas igual a $[12,6$, 24], número de neurônios na camada oculta com possíveis valores de $[25,50,75]$, número de épocas igual a [50,100,150], tamanho de minibatch com possíveis valores de $[75,100]$, a ordem de diferenciação dos dados com possíveis valores de [0, 12]. Em conjunto todas essas possibilidades fazem ao todo uma quantidade de configurações de rede neural de 108, as quais foram repetidas 10 vezes cada por causa da aleatoriedade dos pesos da rede neural. Em outras palavras, para cada configuração de rede neural se executou 10 repetições e o valor final de desempenho foi a média desses 10 valores MAPE.

\section{Modelo CNN}

Para fins de simulação se fixou uma rede neural convolucional de uma camada convolucional com 
função de ativação RELU, função máximo para uma camada pooling, otimizador ADAM, função perda MSE e um neurônio na camada de saída. Os hiperparâmetros que serão buscados no grid search são: número de entradas igual a $[12,6,24]$, número de filtros com possíveis valores de $[32,64]$, tamanho do kernel com possíveis valores de $[3,5]$, número de épocas igual a $[50,100,150]$, tamanho de minibatch com possíveis valores de $[75,100]$, a ordem de diferenciação dos dados com possíveis valores de [0, 12]. Em conjunto todas essas possibilidades fazem ao todo uma quantidade de configurações de rede neural de 144 as quais foram repetidas 10 vezes cada por causa da aleatoriedade dos pesos da rede neural. Em outras palavras, para cada configuração de rede neural convolucional foi executada em 10 repetições e se tomou como valor final de desempenho a média desses 10 valores MAPE.

\section{Modelo LSTM}

Para fins de simulação se fixou uma rede neural LSTM de uma camada oculta com função de ativação RELU, otimizador ADAM, função perda MSE e um neurônio na camada de saída. Os hiperparâmetros que serão buscados no grid search são: número de entradas igual a $[12,6,24]$, número de neurônios na camada oculta com possíveis valores de $[25,50,75]$, número de épocas igual a $[50,100,150]$, tamanho de minibatch com possíveis valores de $[75,100]$, a ordem de diferenciação dos dados com possíveis valores de $[0,12]$. Em conjunto todas essas possibilidades fazem ao todo uma quantidade de configurações de rede neural de: 108, as quais foram repetidas 10 vezes cada por causa da aleatoriedade dos pesos da rede neural. Em outras palavras, para cada configuração de rede neural foi executado 10 repetições e se tomou como valor final de desempenho a média desses 10 valores MAPE.

\subsubsection{Métrica de avaliação}

Para avaliar o desempenho de previsão univariado dos 5 tipos de modelos usados, foi usado o Erro Percentual Médio Absoluto (MAPE). O MAPE pode ser definido com a equação abaixo:

$$
\mathrm{MAPE}=\frac{100 \%}{N} \sum_{i=1}^{N}\left|\frac{y_{i}-\hat{y}_{i}}{y_{i}}\right|
$$

Onde: "N" representa o número de exemplos para treinamento; $y_{i}$ representa o valor atual (real) e $y_{i}$ (com chapéu) representa o valor da previsão dado pelo modelo (clássico ou de aprendizado de máquina).

\subsubsection{Teste de hipótese}

Para testar os resultados das previsões dos modelos clássicos e de machine learning (dois a dois), foi usado o método de teste de significância estatística de Deibold-Mariano [9], com modificação sugerida por Harvey [10] para estatisticamente comparar dois conjuntos de previsões. Adicionalmente, utilizou-se um nível de significância do $5 \%$ (alpha $=0,05$ ) para ser comparado com o valor $\mathrm{p}$ ( $p$-value), o qual é originado através do uso do teste [9] entre dois modelos de previsão e discernir se a diferença associada ao desempenho MAPE entre dois modelos de previsão é estatisticamente significativa ou não.

A continuação é definida nas hipóteses Nula $(\mathrm{Ho})$ e Alternativa ( $\mathrm{Ha}$ ). Cabe dizer que o teste estatístico segue uma distribuição T-Student com grau de liberdade $(T-1)$, onde $T$ é o cumprimento da série temporal.

- Hipótese Nula (Ho): Os algoritmos provavelmente têm o mesmo desempenho.

- Hipótese Alternativa (Ha): Provavelmente existe uma diferença real entre o desempenho médio dos algoritmos.

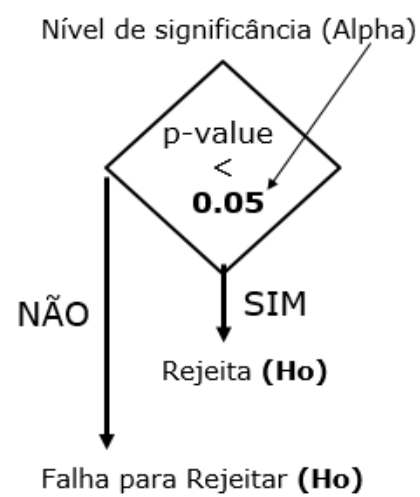

Figura 9. Verificação do $p$-value com o nível de significância (alpha) para rejeição ou aceitação da $\mathrm{H}_{0}$.

\section{Resultados e Discussões}

Os resultados com todas as previsões segundo as melhores configurações de cada modelo estão apresentados na Tabela 2. De todas as possibilidades de configuração e parâmetros que cada um dos modelos poderia adotar, foram escolhidos aquelas configurações e parâmetros que retornaram o menor erro associado (MAPE). 
Cabe lembrar que a previsão diária do Nordeste que consta na Tabela 2, na realidade, é uma média mensal das previsões diárias. Por exemplo, para o caso do modelo MLP associado ao mês de janeiro tem-se o valor de $9788,26 \mathrm{MW}$, o qual expressa o valor médio das 31 previsões médias diárias (lembrar que foram executadas 10 repetições por modelo por causa da estocasticidade dos modelos de machine learning).

Para testar os resultados das previsões dos modelos clássicos e dos modelos de machine learning dois a dois foi utilizado o método de teste de significância estatística de Deibold-Mariano [9], com modificação sugerida por Harvey [10] para que fosse possível comparar estatisticamente dois conjuntos de previsões. Para tanto foi adotado a seguinte sequência de comparação 2 a 2: (MLP v.s. CNN $\rightarrow$ ganhador v.s. LSTM $\rightarrow$ ganhador v.s. SARIMA -> ganhador v.s. SIMPLES).

A escolha pelo modelo mais eficiente se fez quando observado que: i) se no teste de hipótese os modelos possuíam diferenças estatísticas, ou seja, a métrica do erro associado aquele modelo comparado com o erro associado ao outro modelo foram estatisticamente diferentes, optou-se pelo modelo de menor erro MAPE, visto que os modelos não têm a mesma eficácia; ii) se o teste de hipótese dos erros associados aos modelos não possuíssem diferença, estatisticamente significante, ou seja, usar um ou o outro modelo traria a mesma qualidade de previsão, optou-se por continuar escolhendo o modelo com menor MAPE associado.

Desta forma, garantiu-se que o modelo escolhido era o robusto no trabalho de previsão ou por diferença estatística do seu erro associado ou por possuir uma acurácia melhor quando os modelos se mostraram equivalentes. A tabela 2 resume todos os resultados e os valores $p$-value.

A Tabela 3 mostra o modelo escolhido (coluna em destaque) baseado no teste de hipótese de DeiboldMariano. Cabe dizer que o caractere ' $x$ ' significa que não foi necessário comparar esses dois modelos associados já que segundo a sequência adotada só o modelo vencedor é usado para ser validado com o modelo seguinte da sequência.

\section{Conclusões}

Neste trabalho foi proposto uma instigante metodologia de previsão de consumo de energia elétrica utilizando dados temporais do consumo de energia e com auxílio diversas arquiteturas de redes neurais obter a melhor configuração para previsão de um passo temporal à frente.

Outrossim, essa metodologia é facilmente replicada a outros conjuntos de dados ou contextos, onde é possível prever a demanda de uma determinada região, por exemplo: a demanda por energia elétrica de uma unidade federativa em particular ou obter a demanda por um tipo específico de fonte geradora.

Previsões bem-sucedidas orientam planejamentos que balizam investimentos. O setor elétrico é por natureza complexo e intensivo em capital, ou seja, os valores dispendidos são altos e o prazo de execução de novas obras são longos e custosos. Assim quanto mais certeiras forem as previsões de oferta e demanda por energia elétrica, melhores serão as decisões de investimento que beneficiarão as empresas, a população e o próprio Sistema Integrado Nacional - SIN. 
Tabela 2. Previsões dos melhores modelos para região Nordeste.

\begin{tabular}{|c|c|c|c|c|c|}
\hline \multirow{4}{*}{2019} & \multicolumn{5}{|c|}{ Modelos } \\
\hline & MLP & CNN & LSTM & SARIMA & SIMPLES \\
\hline & $\begin{array}{c}{[24,50,} \\
150,75,0]\end{array}$ & $\begin{array}{l}{[24,32,3,} \\
150,75,0]\end{array}$ & $\begin{array}{c}{[6,75,100,} \\
75,0]\end{array}$ & $\begin{array}{l}{[(2,1,1),(2,} \\
\left.1,1,12), c^{2}\right]\end{array}$ & $\begin{array}{c}{[7,1,} \\
\text { 'persist'] }\end{array}$ \\
\hline & \multicolumn{5}{|c|}{ Previsões médias mensais (MW) } \\
\hline Janeiro & 9788,268 & 9840,147 & 10472,735 & 10094,148 & 8963,401 \\
\hline Fevereiro & 10083,585 & 10153,620 & 10221,148 & 9844,530 & 10413,597 \\
\hline Março & 10770,224 & 10591,443 & 10179,315 & 10535,988 & 10776,572 \\
\hline Abril & 11019,022 & 10956,727 & 10147,490 & 10612,122 & 10860,778 \\
\hline Maio & 10721,282 & 10749,177 & 10961,555 & 10145,068 & 10475,655 \\
\hline Junho & 9970,644 & 10183,437 & 10484,975 & 10049,624 & 9806,791 \\
\hline Julho & 10189,435 & 10164,343 & 10616,271 & 9788,138 & 9965,764 \\
\hline Agosto & 10282,126 & 10260,220 & 10867,675 & 10689,474 & 9273,090 \\
\hline Setembro & 11335,340 & 11291,900 & 10828,933 & 10658,803 & 10591,020 \\
\hline Outubro & 11216,488 & 11026,999 & 10757,530 & 11331,754 & 11079,842 \\
\hline Novembro & 11169,583 & 10970,661 & 10768,852 & 11150,502 & 11260,702 \\
\hline Dezembro & 10759,300 & 10858,772 & 10944,219 & 10681,107 & 10752,105 \\
\hline MAPE (\%) & 1,894 & 1,867 & 4,343 & 4,390 & 2,677 \\
\hline
\end{tabular}

Tabela 3. Teste de hipótese de 2 em 2 para os melhores modelos para região Nordeste.

\begin{tabular}{cccccc}
\hline \multirow{2}{*}{ p-value } & \multicolumn{5}{c}{ Modelos } \\
\cline { 2 - 6 } & MLP & CNN & LSTM & SARIMA & SIMPLES \\
\hline MLP & - & 0,107 & $\mathrm{x}$ & $\mathrm{x}$ & $\mathrm{x}$ \\
\hline CNN & 0,107 & - & 0,244 & 0,232 & 0,715 \\
\hline LSTM & $\mathrm{x}$ & 0,244 & - & $\mathrm{x}$ & $\mathrm{x}$ \\
\hline SARIMA & $\mathrm{x}$ & 0,232 & $\mathrm{x}$ & - & $\mathrm{x}$ \\
\hline SIMPLES & $\mathrm{x}$ & 0,715 & $\mathrm{x}$ & $\mathrm{x}$ & - \\
\hline
\end{tabular}

\section{Referências}

[1] EPE - EMPRESA DE PESQUISA ENERGÉTICA. Resenha mensal do mercado de energia elétrica Ano XII, Número 136, janeiro de 2019. Disponível em: $<$ https://www.epe.gov.br/pt>. Acessado em 20/07/2020.

[2] BEZERRA, F. D. Panorama da Infraestrutura no Nordeste do Brasil: Energia Elétrica. In: Caderno Setorial ETENE, Ano 4, no 65. Jan2019.

[3] BROWNLEE, J. Deep learning for time series forecasting: predict the future with MLPs, CNNs and LSTMs in python. 2019.

[4] BROWNLEE, J. Introduction to time series forecasting with python: discover how to prepare data and develop models to predict the future. 2019.

[5] BOX, G.E.P., JENKINS, G.M., REINSEL, G.C., LJUNG, G.M. Time series analysis: forecasting and control. Wiley. 2016.
[6] PEDRO, H F N. Previsão de séries temporais utilizando uma combinação nãolinear entre ARIMA e Redes Neurais Artificiais. 2017.

[7] VARGAS, A. C. G.; PAES, A. e VASCONCELOS, C. N. Um Estudo sobre Redes Neurais Convolucionais e sua Aplicação em Detecção de Pedestres. 2016.

[8] BROWNLEE, J. Long short-term memory networks with python: develop deep learning models for your sequence prediction problems. 2019.

[9] DEIBOLD, F. X; MARIANO, R.S. Comparing Predictive Accuracy. Journal of Business and Economic Statistics. Vol 3, N 3. 1995.

[10] HARVEY, D; LEYBOURNE, S; NEWBOLD, $P$. Testing the equality of prediction mean squared errors. International Journal of Forecasting. 1997. 